\title{
Editorial: Biomarkers in Neurology
}

\author{
Stefania Mondello ${ }^{\text {* }}$, Mohamed Mosaad Salama ${ }^{2}$, Wael M. Y. Mohamed ${ }^{3,4}$ and \\ Firas H. Kobeissy ${ }^{5,6}$
}

${ }^{1}$ Department of Biomedical and Dental Sciences and Morphofunctional Imaging, University of Messina, Messina, Italy, ${ }^{2}$ Institute of Global Health and Human Ecology, American University in Cairo, Cairo, Egypt, ${ }^{3}$ Clinical Pharmacology Department, Menoufia Medical School, Menoufia University, Al Minufya, Egypt, ${ }^{4}$ Department of Basic Medical Science, Kulliyyah of Medicine, International Islamic University, Kuantan, Malaysia, ${ }^{5}$ Department of Emergency Medicine, University of Florida, Gainesville, FL, United States, ${ }^{6}$ Department of Biochemistry and Molecular Genetics, Faculty of Medicine, American University of Beirut, Beirut, Lebanon

Keywords: biomarkers, neurological disorders \& brain damage, diagnostic test, prognosis, neurodegeneration, brain Injury - Traumatic

\section{Editorial on the Research Topic}

\section{Biomarkers in Neurology}

Neurological disorders constitute a major health and socioeconomic problem. They represent the second cause of death and the leading cause of disability throughout the world. Despite the implementation of strategies and intervention programs to reduce the burden, over the past 25 years, the incidence, prevalence, mortality, and disability rates of neurological disorders are rising globally, mainly due to population aging and growth (1). This has placed heavy pressure on health-care systems pointing out the urgent need to identify new strategies to improve patient outcomes and reduce health costs by enabling more effective drug development and establishing a more personalized medicine approach.

Rapid scientific and technical advances have enabled reliable and affordable measurement

Edited and reviewed by: Mark P. Burns,

Georgetown University, United States

*Correspondence:

Stefania Mondello stm_mondello@hotmail.com

Specialty section: This article was submitted to Neurodegeneration, a section of the journal Frontiers in Neurology

Received: 27 January 2020 Accepted: 28 February 2020 Published: 18 March 2020

Citation:

Mondello S, Salama MM Mohamed WMY and Kobeissy FH (2020) Editorial: Biomarkers in Neurology. Front. Neurol. 11:190. doi: 10.3389/fneur.2020.00190 of novel biomarkers-biological indicators that objectively measure and evaluate physiological or pathophysiological processes or pharmacological responses to a therapeutic intervention (2) - which have been suggested to help assessment and management of patients with neurological disorders beyond current practice standards (3-5). Evidence suggests a potential variety of clinical applications, including enhancing diagnostic and prognostic accuracy, improving the existing decision criteria for early diagnosis and risk stratification, as well as assisting in disease monitoring, and acting as surrogate endpoints in experimental studies and clinical trials (6-10). In addition, biomarkers may reliably capture the different aspects of disease heterogeneity and pathogenesis, helping characterize patients, and thereby informing targeted tailored treatments and predicting response outcomes to interventions (11-18). However, despite large numbers of candidate biomarkers have been proposed and extensively evaluated, very few are currently integrated into routine clinical practice and the quest for novel brain injury markers in still ongoing (19).

This book aimed at providing an overview of the biomarker landscape in neurological disorders. The diverse authors discuss established and emerging biomarkers as well as innovative strategies for identifying novel candidates offering new and unique perspectives. Several articles in this volume have been focused on Alzheimer's disease and other neurodegenerative disorders, exploring potentially relevant genetic signature (Chen et al.) and the pathogenetic and prognostic role of circulating cytokines (Kim et al.). Importantly, using a methodologically novel approach that 
combines computational prediction and experimental validation, Yao et al., for the first time, identified VLDLR, an apolipoprotein E receptor involved in synaptic plasticity, as a circulating signature for Alzheimer's disease. Accordingly, several lines of evidence are pointing toward the added and complementary value of markers of synaptic function owing to their close link with cognitive deterioration (20).

Contemporary investigations on microRNAs (miRNAs) (Di Pietro et al.) and high mobility group box protein 1 (HMGB1) (Paudel et al.) are also presented, highlighting the fact that these markers may be risk factors themselves and therefore potential targets of therapy (21). Diverse contributions recognize the urgent need for reliable diagnostic and prognostic biomarkers in peripheral demyelinating diseases (Kamil et al.) and spinal cord injury (Albayar et al.), with an emphasis on recent advances in medical knowledge and practice; while other work provides an opportunity to study established markers, such as neurofilament light chain, in neonatal neuronal injury (Depoorter et al.), and to demonstrate the theragnostic potential-capability to identify and monitor the drug effect on the molecular pathologyof PAS-positive vacuolated lymphocytes in late-onset Pompe disease patients treated with ERT (Parisi et al.). Finally, the role of lipidomic analysis (Sabogal-Guáqueta et al.) and Fouriertransform infrared imaging spectroscopy and Laser ablation LAICPMS techniques (Ali et al.) in biomarker discovery is outlined.

Overall, this volume offers a unique opportunity to foster knowledge and innovation in the arena of biomarkers for neurological disorders, while stimulating testable hypotheses and

\section{REFERENCES}

1. GBD 2016 Neurology Collaborators. Global, regional, and national burden of neurological disorders, 1990-2016: a systematic analysis for the Global Burden of Disease Study 2016. Lancet Neurol. (2019) 18:459-80. doi: 10.1016/S1474-4422(18)30499-X

2. Biomarkers Definitions Working Group. Biomarkers and surrogate endpoints: preferred definitions and conceptual framework. Clin Pharmacol Ther. (2001) 69:89-95. doi: 10.1067/mcp.2001.113989

3. Mondello S, Hayes RL. Biomarkers. Handb Clin Neurol. (2015) 127:245-65. doi: 10.1016/B978-0-444-52892-6.00016-7

4. Perry LA, Lucarelli T, Penny-Dimri JC, McInnes MD, Mondello S, Bustamante A, et al. Glial fibrillary acidic protein for the early diagnosis of intracerebral hemorrhage: systematic review and meta-analysis of diagnostic test accuracy. Int J Stroke. (2019) 14:390-9. doi: 10.1177/1747493018806167

5. Mondello S, Kobeissy F, Mechref Y, Zhao J, Talih FR, Cosentino F, et al. Novel biomarker signatures for idiopathic REM sleep behavior disorder: a proteomic and system biology approach. Neurology. (2018) 91:e1710-5. doi: 10.1212/WNL.0000000000006439

6. Olsson B, Lautner R, Andreasson U, Ohrfelt A, Portelius E, Bjerke M, et al. CSF and blood biomarkers for the diagnosis of Alzheimer's disease: a systematic review and meta-analysis. Lancet Neurol. (2016) 15:673-84. doi: 10.1016/S1474-4422(16)00070-3

7. Mondello S, Shear DA, Bramlett HM, Dixon CE, Schmid KE, Dietrich $\mathrm{WD}$, et al. Insight into pre-clinical models of traumatic brain injury using circulating brain damage biomarkers: operation brain trauma therapy. J Neurotrauma. (2016) 33:595-605. doi: 10.1089/neu.2015. 4132

8. Hellewell SC, Mondello S, Conquest A, Shaw G, Madorsky I, Deng JV, et al. Erythropoietin does not alter serum profiles of neuronal and axonal biomarkers after traumatic brain injury: findings from the the development of a strategic research agenda to accelerate their incorporation into routine clinical practice.

\section{AUTHOR CONTRIBUTIONS}

SM wrote the original draft, assembled and incorporated comments from the co-authors and crafted the final draft. All of the other co-authors contributed to manuscript review and revision.

\section{FUNDING}

SM and FK received research support from the Italian Ministry of Health (GR-2013-02354960).

\section{ACKNOWLEDGMENTS}

We thank our colleagues for devoting their time, expertise and effort in producing valuable contributions that provide rigorous frameworks and innovative and critical insights. This book would not have been possible without their important work. It is also a pleasure to acknowledge and thank the editorial team for their expert assistance and support in the development and production of this volume. Finally, with our utmost gratitude and profound respect, we want to dedicate this book to all patients and their families. We are indebted to their sacrifices and unfailing spirit of hope which represent our greatest source of inspiration.
Australian EPO-TBI clinical trial. Crit Care Med. (2018) 46:554-61. doi: 10.1097/CCM.0000000000002938

9. Parnetti L, Gaetani L, Eusebi P, Paciotti S, Hansson O, El-Agnaf O, et al. CSF and blood biomarkers for Parkinson's disease. Lancet Neurol. (2019) 18:573-86. doi: 10.1016/S1474-4422(19)30024-9

10. Czeiter E, Mondello S, Kovacs N, Sandor J, Gabrielli A, Schmid K, et al. Brain injury biomarkers may improve the predictive power of the IMPACT outcome calculator. J Neurotrauma. (2012) 29:1770-8. doi: 10.1089/neu.201 1.2127

11. Mondello S, Papa L, Buki A, Bullock MR, Czeiter E, Tortella FC, et al. Neuronal and glial markers are differently associated with computed tomography findings and outcome in patients with severe traumatic brain injury: a case control study. Crit Care. (2011) 15:R156. doi: 10.1186/cc10286

12. Ren C, Kobeissy F, Alawieh A, Li N, Li N, Zibara K, et al. Assessment of serum UCH-L1 and GFAP in acute stroke patients. Sci Rep. (2016) 6:24588. doi: $10.1038 /$ srep 24588

13. Mondello S, Palmio J, Streeter J, Hayes RL, Peltola J, Jeromin A. Ubiquitin carboxy-terminal hydrolase L1 (UCH-L1) is increased in cerebrospinal fluid and plasma of patients after epileptic seizure. BMC Neurol. (2012) 12:85. doi: 10.1186/1471-2377-12-85

14. Constantinescu R, Mondello S. Cerebrospinal fluid biomarker candidates for parkinsonian disorders. Front Neurol. (2012) 3:187. doi: $10.3389 /$ fneur.2012.00187

15. Mondello S, Jeromin A, Buki A, Bullock R, Czeiter E, Kovacs N, et al. Glial neuronal ratio: a novel index for differentiating injury type in patients with severe traumatic brain injury. J Neurotrauma. (2012) 29:1096-104. doi: 10.1089/neu.2011.2092

16. Ehrenreich H, Kastner A, Weissenborn K, Streeter J, Sperling S, Wang KK, et al. Circulating damage marker profiles support a neuroprotective effect of erythropoietin in ischemic stroke patients. Mol Med. (2011) 17:1306-10. doi: 10.2119/molmed.2011.00259 
17. Frankel M, Fan L, Yeatts SD, Jeromin A, Vos PE, Wagner AK, et al. Association of very early serum levels of S100B, glial fibrillary acidic protein, ubiquitin C-terminal hydrolase-L1, and spectrin breakdown product with outcome in ProTECT III. J Neurotrauma. (2019) 36:2863-71. doi: $10.1089 /$ neu. 2018.5809

18. Mondello S, Kobeissy F, Vestri A, Hayes RL, Kochanek PM, Berger RP. Serum concentrations of ubiquitin C-terminal hydrolase-L1 and glial fibrillary acidic protein after pediatric traumatic brain injury. Sci Rep. (2016) 6:28203. doi: $10.1038 /$ srep 28203

19. Mondello S, Thelin EP, Shaw G, Salzet M, Visalli C, Cizkova D, et al. Extracellular vesicles: pathogenetic, diagnostic and therapeutic value in traumatic brain injury. Expert Rev Proteomics. (2018) 15:451-61. doi: 10.1080/14789450.2018.1464914

20. Blennow K, Zetterberg $H$. The past and the future of Alzheimer's Disease fluid biomarkers. J Alzheimers Dis. (2018) 62:1125-40. doi: 10.3233/JAD-170773
21. Saugstad JA. MicroRNAs as effectors of brain function with roles in ischemia and injury, neuroprotection, and neurodegeneration. J Cereb Blood Flow Metab. (2010) 30:1564-76. doi: 10.1038/jcbfm.2010.101

Conflict of Interest: The authors declare that the research was conducted in the absence of any commercial or financial relationships that could be construed as a potential conflict of interest.

Copyright $\odot 2020$ Mondello, Salama, Mohamed and Kobeissy. This is an open-access article distributed under the terms of the Creative Commons Attribution License (CC $B Y)$. The use, distribution or reproduction in other forums is permitted, provided the original author(s) and the copyright owner(s) are credited and that the original publication in this journal is cited, in accordance with accepted academic practice. No use, distribution or reproduction is permitted which does not comply with these terms. 